\title{
Spectrum analysis of short-period $K$ index behaviour at high and mid-latitudes
}

\author{
P. B. Kotzé \\ South African National Space Agency (SANSA), Space Science, P.O. Box 32, Hermanus 7200, South Africa \\ Correspondence to: P. B. Kotzé (pkotze@sansa.org.za)
}

Received: 8 September 2014 - Revised: 25 November 2014 - Accepted: 2 December 2014 - Published: 9 January 2015

\begin{abstract}
Geomagnetic activity levels during the declining phase and solar minimum period of the solar cycle are considerably different from those during the solar maximum phase. Previous studies revealed variations in the pattern of recurrent activity from cycle to cycle as well as variations in the average geomagnetic activity levels during a solar cycle. During the declining phase of a solar cycle (and solar minimum), the solar and interplanetary causes of geomagnetic activity are substantially different from those during the solar maximum phase. Co-rotating fast solar wind streams originating from large polar coronal holes, extending towards the Sun's equator, interact with the Earth's magnetosphere, resulting in recurrent geomagnetic activity particularly during solar cycle minimum periods. This is a wellknown phenomenon with respect to 27.0- and 13.5-day recurrence geomagnetic activity, and it is well-known to be related to sectorial (non-axial) poloidal magnetic field structure in the Sun. Published results of the recent solar-cycle23 minimum showed that the presence of 9.0- and 6.7-day recurrent geomagnetic activities can be attributed to the sectorial spherical harmonic structure present in the solar magnetic field. In this study we performed a wavelet and LombScargle analysis of the geomagnetic activity $K$ index at Lerwick (LER), Hermanus (HER) and Canberra (CNB) for the period between 1960 and 2010, overlapping with solar cycles 20 to 23 . Daily mean $K$ indices are used to identify how several harmonics of the 27.0-day recurrent period change during each solar cycle when comparing high and mid-latitude geomagnetic activity, applying a $95 \%$ confidence level. In particular the behaviour of the second (13.5-day), third (9.0day) and fourth (6.7-day) harmonics are investigated by doing a wavelet analysis of each individual year's $K$ indices at each location. Results obtained show that particularly during solar minima the 27.0-day period is no longer detectable
\end{abstract}

above the $95 \%$ confidence level, and that geomagnetic activity is in fact dominated by higher harmonics like 13.5-, 9.0and 6.7-day periods. These findings in fact are in line with previous investigations and confirm the results obtained by researchers using other geomagnetic activity indices like $a a$ and C9. The wavelet-spectrum analysis also reveals that during the downward phase of cycle 23 and the very long minimum of 23-24 between 2002 and 2008, the 27.0-day activity period drops below the $95 \%$ confidence level. This is confirmed by Lomb-Scargle analyses of every year's $K$ index activity. Results obtained in this study support evidence by other investigations that this can be attributed to the lack of coronal-mass ejection (CME)-dominated solar activity during solar minima, periods characterized by strong solar dipolar magnetic fields, less sunspot numbers than at solar maxima, and multiple prominent co-rotating solar wind streams present. This analysis further confirms previous studies by other authors that the pattern of recurrent activity is dictated by the configuration of coronal holes which give rise to related high-speed streams during a solar cycle by analysing $K$ indices at both high- and mid-latitude magnetic observatories.

Keywords. Magnetospheric physics (solar-windmagnetosphere interactions)

\section{Introduction}

Solar cycles are characterized by changing recurrent geomagnetic activity which varies with the strength of the solar magnetic field and the emergence of sunspots; e.g. during solar maxima the Sun's magnetic field is primarily toroidal, which changes gradually during the declining phase of the solar cycle to a poloidal dipolar field (e.g. Solanki et al., 


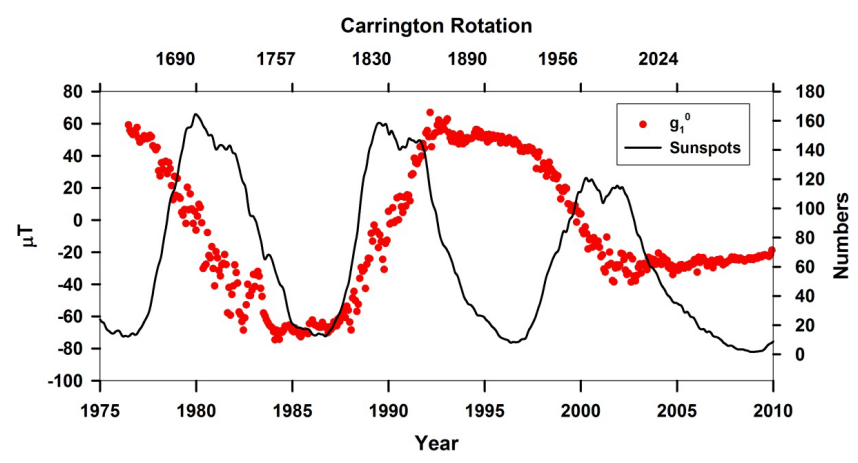

Figure 1. A plot showing the axial dipolar spherical harmonic solar magnetic field $g_{1}^{0}$ coefficient as well as the smoothed monthly mean sunspot numbers between 1975 and 2010, and Carrington rotations 1690-2024.

2006; Love et al., 2012). This results in the gradual disappearance of sunspots and associated coronal-mass ejection (CME)-driven solar activity, with the appearance of large areas of open magnetic flux accompanied by coronal-holedriven solar activities during solar minimum, while the value of the dipolar solar magnetic field also fluctuates during a solar cycle (Legrand and Simon, 1991), as shown in Fig. 1 for the axial dipole spherical harmonic coefficient $g_{1}^{0}$, reaching very small values during solar maximum, and maximum strength during solar minimum. Past studies have shown that the dominant periodicity for geomagnetic activity recurrence is a 27.0-day period (Maunder, 1905; Bartels, 1932; Cliver, 1995; Tsurutani et al., 2006). Using a wavelet analysis, Torrence and Compo (1998) showed that the power of the recurrent activity in the C9 index during 1950-2004 varies substantially during a particular solar cycle. In fact they showed that the temporal pattern of power clearly varies from cycle to cycle, and that intermittent 27.0- and 13.5-day periods, corresponding to one- or two-component co-rotating streams, are evident. Torrence and Compo (1998) also showed that the 27.0-day recurrent activity was unusually weak during the mid-1960's solar minimum. In a recent study by Love et al. (2012) the following question was addressed: to what extent do other harmonics also play a role during various phases of different solar cycles, and if a possible correlation exists with recurrent solar magnetic field variations, particularly during periods of sunspot minimum as has been experienced for the years during minimum 23-24? During 2008, fewer sunspots have been detected than in any year since 1913. Studies on solar wind data in 2005 show 9.0- and 6.7-day recurrence periods (Temmer et al., 2007), while the same periods, corresponding to the $3 \mathrm{rd}$ and 4 th harmonics of the fundamental 27.0-day solar rotation period, could also be identified in geomagnetic activity (Lei et al., 2008) for years around solar cycle minimum 23-24.

In this investigation a wavelet signal processing method was used to analyse the $K$ index geomagnetic activity variations at Lerwick $\left(60.13^{\circ},-1.18^{\circ}\right)$, a high-latitude auro- ral magnetic observatory, as well as Hermanus $\left(-34.425^{\circ}\right.$, $\left.19.225^{\circ}\right)$ and Canberra $\left(-35.32^{\circ}, 149.36^{\circ}\right)$, two mid-latitude observatories. This was done in order to determine similarities and differences in the behaviour of the different harmonics (13.5-, 9.0- and 6.7-day) of the fundamental 27.0-day solar rotation during the time interval between 1960 and 2010 which includes four solar cycles. The annual time series, based on daily mean $K$ indices, show strong non-stationary periodic behaviour where the different harmonics appear intermittently with varying strengths. In addition, the LombScargle (Lomb, 1976; Scargle, 1982) method is also employed for comparison purposes.

\section{Data}

The $3 \mathrm{~h} K$ index data for Canberra (CNB) were downloaded (http://www.ga.gov.au/oracle/geomag) for the period 1985-2010 and then used to calculate daily mean values for each year. A complete record of 9496 days with no missing days was obtained for CNB. In the case of LER, data were retrieved from the British Geological Survey web page (http://www.geomag.bgs.ac.uk/data_service/data) for the period 1960-2010, with only 28 days of missing data out of a total of 18628 days. HER data were not available for 1962 , while in the case of 1974 , too many data gaps consisting of several days at a time were present, rendering this year unsuitable for any analysis. A total record of 17898 days of which 52 days contained no data could be compiled for HER. Daily means were only determined when more than $90 \%$ of data were available, and in the case of missing daily mean values spline interpolation was used to fill single day gaps. For both Hermanus (HER) and Lerwick (LER) the missing days consisted of single days scattered randomly between 1960 and 2010. The spline interpolation subsequently did not have any influence on the spectral analysis results. The Wilcox Solar Observatory solar daily mean magnetic field data between 1976 and 2010 were also obtained (http://wso.stanford.edu/), but as too many consecutive days were sometimes encountered with missing data, no interpolation was applied; therefore it was regarded as an irregular time series. All graphs and plots in this paper were done with the plotting package SigmaPlot (www.systat.com), while the contour plot in Fig. 5 was the result of output generated using the Interactive Data Language (IDL) (http://www. exelisvis.com/ProductsServices/IDL.aspx) wavelet applet.

\section{Wavelet and Lomb-Scargle analysis}

Wavelet analysis is a powerful tool to decompose a time series into time-frequency space and to determine both the dominant modes of variability, in particular when these time series contain non-stationary power at different frequencies (Daubechies, 1992). In this investigation a Morlet wavelet (Morlet et al., 1982; Torrence and Compo, 1998), $\varphi(t)$ con- 
sisting of a plane wave modulated by a Gaussian, was applied:

$\varphi(t)=t^{-1 / 4} e^{i \omega_{0} t} e^{-t / 2}$,

where $\omega_{0}$ is the non-dimensional frequency, and while $t$ is the non-dimensional time parameter. Wavelets allow the analysis of non-stationary signals to investigate the periodicity content and localization in time or space. This space/time localization is possible as the wavelet function $\varphi$ is defined in a finite interval. In wavelet analysis it is therefore possible to represent signals $f(t)$ by series such as follows:

$f(t)=\sum_{j=-\infty}^{\infty} \sum_{k=-\infty}^{\infty} c_{k}^{j} \varphi_{k}^{j}(t)$,

where $\varphi_{k}^{j}=\varphi\left(2^{j} t-k\right)$ are called the mother wavelets (Morlet in this investigation) and $c_{k}^{j}$ are the wavelet coefficients, whose amplitude (power) are directly proportional to the signal they represent. For the purposes of this investigation Morlet orthogonal wavelet functions of order 5 were chosen, as they are symmetric and provide an effective representation of high-order polynomials, which are suited for the detection of short-period behaviour in $K$ index time series consisting of daily mean values. Wavelet transforms were also employed by Balasis et al. (2006) to perform a fractal spectral analysis of the 2001 Disturbance storm-time (Dst) index time series at solar maximum.

The Lomb-Scargle method on the other hand was used to verify results obtained from wavelet analysis. This spectrum analysis method, being a variant of the Fourier transform with particular application to unevenly sampled data, represents a signal as the sum of sine and cosine functions of infinite duration. The computer algorithm (Press et al., 1992, chapter 13.8) is applied to the daily mean values for each year between 1960 and 2010. In the case of our $K$ indices the statistical properties and characteristic periodicities vary with time, so we could only obtain information about the frequency content without any localization in time.

\section{Results and discussion}

The wavelet power spectra obtained by analysing the daily mean $K$ indices at LER and HER for each individual year between 1960 and 2010 inclusive, made it possible to identify periodic components to a confidence level of $95 \%$. In particular the short-term periodicities corresponding to the 2 nd, $3 \mathrm{rd}$, and 4 th harmonics of the fundamental solar rotation period of 27.0 days were investigated during each solar cycle in this time interval. In the case of CNB, data without gaps of several consecutive days for $K$ indices were only available between 1985 and 2010, and allowed a comparison between CNB and HER, located at mid-latitudes, as well as LER situated at high-latitudes, for this particular time interval.
LER K Wavelet Periods
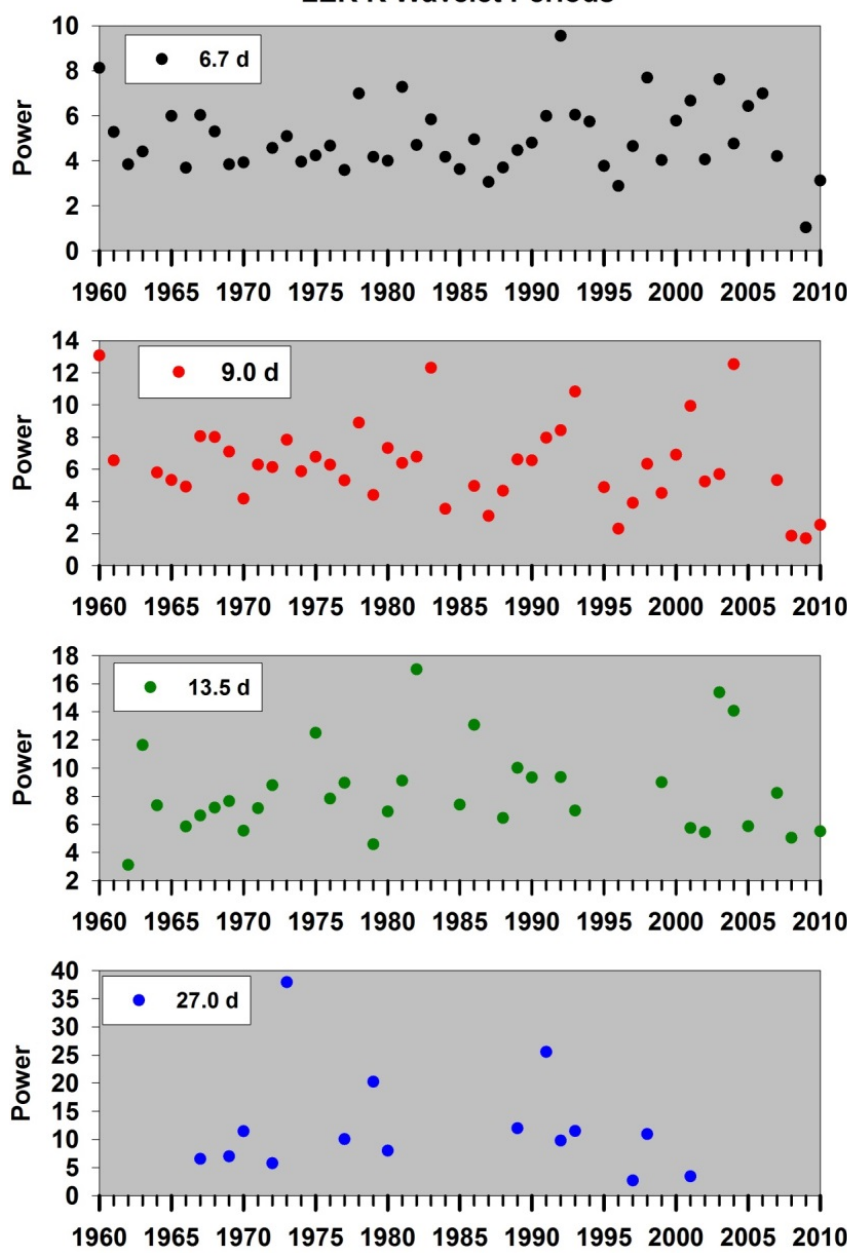

Figure 2. Recurrence periods as identified by wavelet analysis of LER annual time series $K$ indices from 1960 until 2010.

In order to determine the short-period $K$ index geomagnetic activity recurrence, a Morlet wavelet analysis was performed for each year between 1960 and 2010 for both LER and HER, while in the case of CNB the time interval was between 1985 and 2010. This enabled the identification of 6.7-, 9.0-, 13.5- and 27.0-day periods in the annual data intervals at a confidence level of $95 \%$, and particularly the behaviour of these periodicities during the various solar cycles investigated. Results obtained for LER, HER and CNB are shown in Figs. 2-4.

A power spectrum for LER is shown in Fig. 5 for 2007 when solar activity was extremely low, showing that the short periods and particularly the 13.5-day periodicities are the most prominent.

From these figures it is quite clear that the different periodicities appear without a fixed pattern during each year and that the average power scales according to the particular harmonic. During the time interval particularly between 2002 and 2009, the power spectra at LER, HER and CNB 
HER K Wavelet Periods
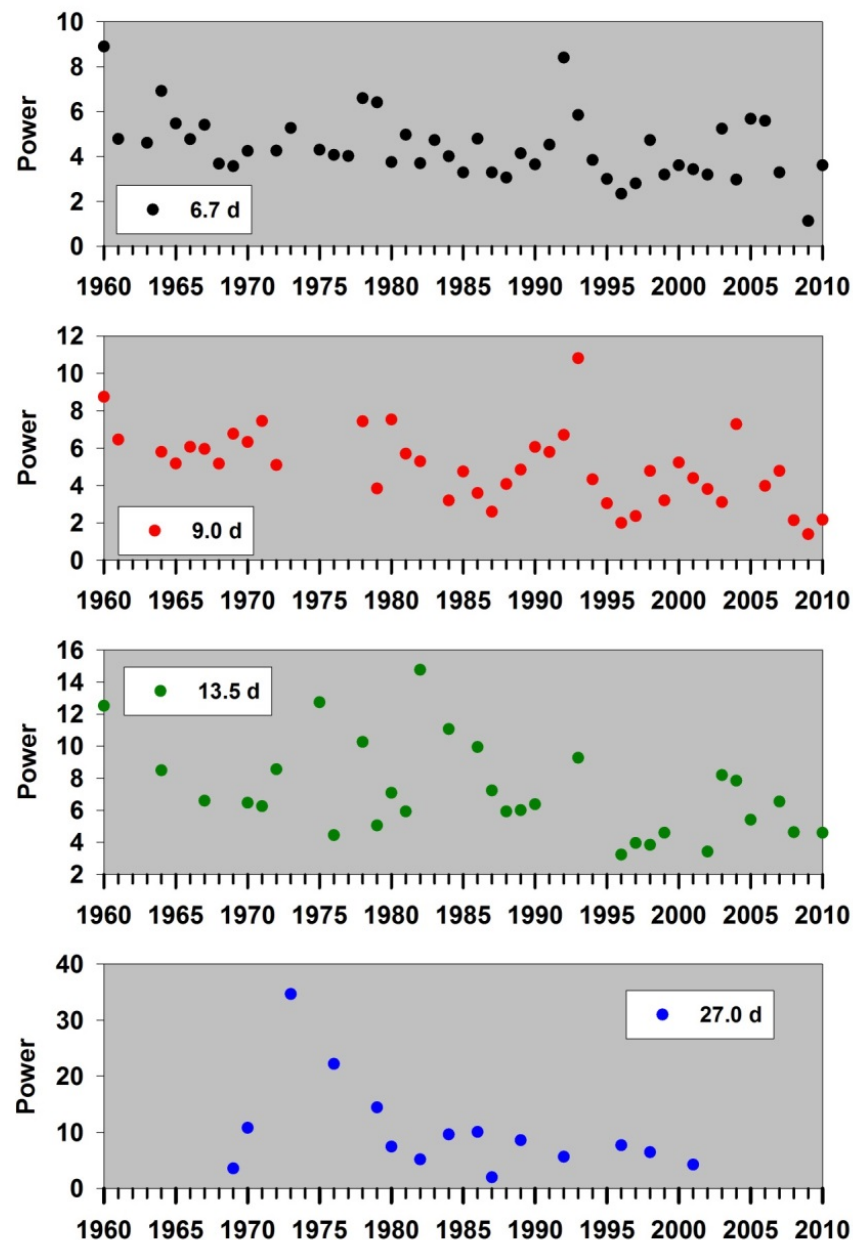

Figure 3. HER recurrence periods as identified in the annual time series of $K$ indices using wavelets from 1960 until 2010.

are dominated by the 13.5-day period, with the 27.0-day period below the confidence level of $95 \%$. This phenomenon is indeed also observed during and around solar cycle minima occurring at 1965 and 1976 . This is possibly due to magnetic storms that result from the sporadic occurrence of coronal-mass ejections, and the fact that fast streams from coronal holes during solar cycle minima are more dominant. This finding is in line with results obtained by Katsavrias et al. (2012) who found that the 27.0-day period could not be detected at a confidence level of $99 \%$ during the minimum of cycle 23 in the case of the interplanetary magnetic field components. It has been proposed in previous investigations of periodicities in solar wind parameters (e.g. Mursula and Zieger, 1996) that activities dominated by a 13.5 -day periodicity are due to the occurrence of two high-speed streams per solar rotation. On the other hand the power of the 6.7-day periodicity during solar minima is on average $30 \%$ stronger than during solar maxima for the period stretching from 1965 until 2010 as shown by the behaviour observed at LER and
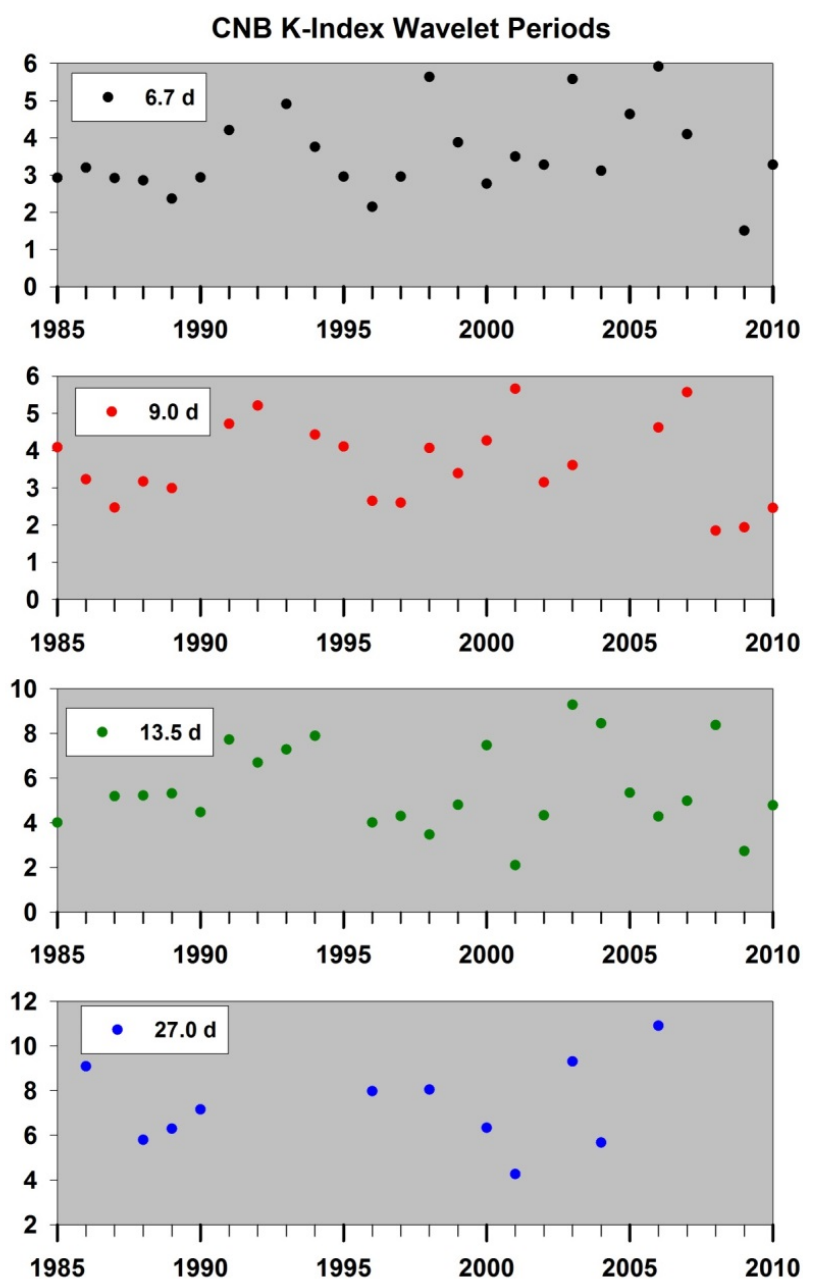

Figure 4. Periods identified in the annual time series of $K$ indices as recorded at CNB between 1985 and 2010 using a Morlet wavelet analysis.

HER. In fact during 2006 the 6.7-day period is the dominating periodicity above a confidence level of $95 \%$ at both LER, HER, and CNB, as revealed by a Lomb-Scargle analysis. The 27.0-day period could not be clearly identified, and only appears as a broad peak stretching from 26 to 30 days during 2006 below the $95 \%$ confidence level. A Lomb-Scargle spectrum analysis of the solar magnetic field in 1995, 2005 and 2006 revealed that only the 13.5-day period is present, suggesting a correlation between solar magnetic field and geomagnetic activity periodicity as shown by $K$ index behaviour, particularly during solar cycle minimum when it is observed that the 27.0-day period appears to be less significant as revealed by both wavelet and Lomb-Scargle analysis. Several publications have appeared over the years on results obtained by studying the behaviour and influence of solar cycle and solar wind parameters on geomagnetic activity levels (e.g. Crooker et al., 1977; Kojima and Kakinuma, 1990; Mursula, 1999), periodicities in the interplanetary magnetic field 


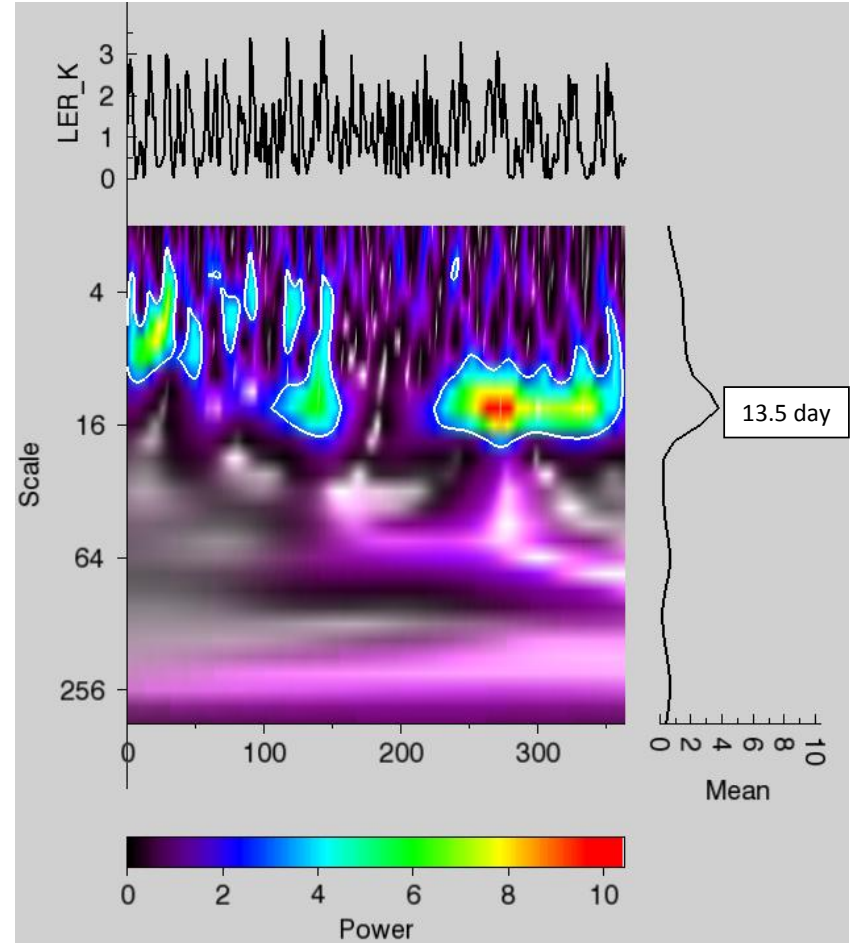

Figure 5. Morlet wavelet $K$ index power spectrum for LER during 2007 showing the periodicities at a confidence level of $95 \%$ (indicated by the white contour lines). The absence of the 27.0-day geomagnetic-activity recurrence interval is to be noted.

(Gonzalez and Gonzalez, 1987), and solar excursion phases during several solar cycles (Mursula and Zieger, 1998). Results obtained by these and other authors showed that the origin of periodicities in geomagnetic activities is linked to the domination of solar activity during solar cycle minimum by coronal holes (e.g. Sheeley Jr. et al., 1976), responsible for multiple-component high-speed streams, while during solar maximum CMEs are the main drivers of geomagnetic activity (Gosling et al., 1991). It is also known that the structure of the heliosheet is controlled by the strength of the solar dipole configuration, i.e. during sunspot maximum intervals, the heliosheet has a complex non-dipolar structure, controlled by sectorial solar magnetic fields, as shown by Love et al. (2012). The occurrence rate of CME-related storms has a tendency to follow the sunspot cycle with large magnetic storms occurring during solar maxima (Richardson et al., 2001).

Figure 1 shows plots of monthly mean values of both the axial dipole spherical harmonic coefficient $g_{1}^{0}$ (Wilcox Observatory models at solar radius $R_{\mathrm{S}}=2.5$ ) and sunspot numbers between 1975 and 2010 and Carrington rotations 16902024. From these plots, it is clear that also the axial dipolar solar magnetic field behaved differently during the minimum of 23-24, particularly between 2003 and 2010. Instead of reaching a maximum value like in the previous solar cy-
Lomb-Scargle: $2008 \mathrm{~K}$ indices
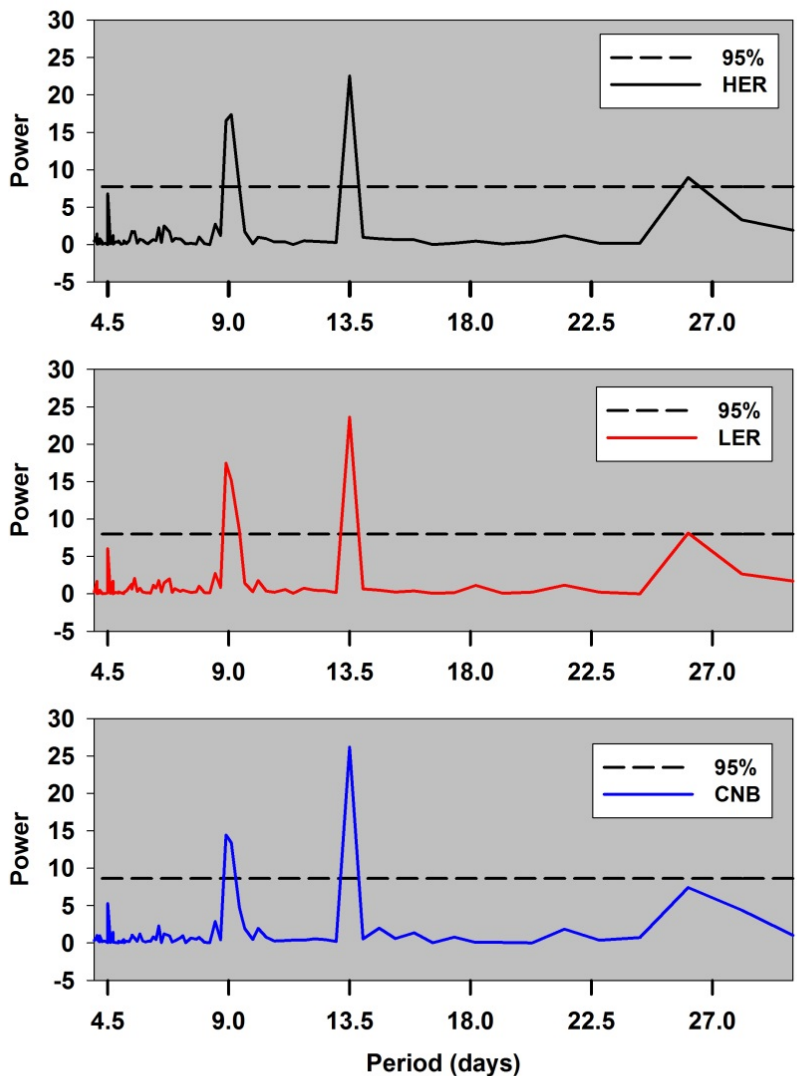

Figure 6. A Lomb-Scargle analysis of the periodicity at HER, LER and CNB during 2008 showing the dominance of the 9.0- and 13.5day periods above the $95 \%$ confidence level.

cles, $g_{1}^{0}$ stayed at a relatively low level, while the sunspot numbers diminished to a very low minimum. This compliments the finding of Love et al. (2012) that the solar dynamo reached a state of unusual asymmetry during the recent minimum 23-24, resulting in a non-axisymmetric heliosheet that led to the strong 9.0- and 6.7-day geomagnetic activity recurrence.

The 9.0- and 13.5-day periodicity seem to play an important and distinguishable role particularly during the downward phase and minimum periods of several solar cycles as revealed in this investigation. This was quite evident during 1985, 1994, 1996 and 2008 at all three observatories, but not around 1975 at HER, the minimum of cycle 20. A LombScargle analysis confirmed the wavelet analysis results. In particular for 2008, as shown in Fig. 6, only the 9.0- and 13.5-day periods can be distinguished above the $95 \%$ level. Although a 26- and 28-day periodicity can be identified in the spectrum of the solar magnetic field during 2008, the solar activity is dominated by the 13.5-day period. In fact during 2005 and 2006 only the 13.5-day periodicity can be seen in the solar magnetic field spectrum, while in 2009 the 27.0-day 
periodicity has recovered its power and is starting to be the most dominant period, and in the process coinciding with the rising period of cycle 24 .

\section{Conclusions}

Several interesting features regarding the behaviour of the different harmonics of the 27.0-day synodic rotation of the Sun have been revealed by this wavelet and Lomb-Scargle spectrum analysis of $K$ indices at both high and mid-latitudes during 1960-2010, as observed at LER, HER, and CNB. Of particular interest is that recurrent geomagnetic activity changes over the time interval of each solar cycle, particularly the 6.7-, 9.0-, 13.5-day and also the 27.0-day periods. At solar cycle maximum, the dominant interval for geomagnetic activity recurrence is a 27.0-day period, coinciding with low solar magnetic field strength and maximum sunspot numbers. During solar maximum periods, solar activity is predominantly characterized by increased levels of coronalmass ejections, which act as the main drivers for the observed increase of geomagnetic activity (Gosling et al., 1991; Cliver, 1995; Richardson et al., 2001). At solar minimum on the other hand, the solar activity conditions are characterized by low sunspot numbers, with semi-isolated coronal holes emitting high-speed solar wind streams (Neupert and Pizzo, 1974), responsible for lower geomagnetic activity as reflected by $K$ index behaviour. The study by Richardson et al. (2001) concluded that storms associated with streams are most prevalent during the decay and minimum phase of the solar cycle. These storms are generally small or medium in size in comparison to solar maximum conditions. The declining phase of cycle 23 and the following very low and unusually long minimum of 23-24 were different than previous solar cycles (e.g. Russell et al., 2010). Studies for the years near minimum 23-24 showed high levels of semi-persistent 9.0and 6.7-day recurrence intervals in solar wind data (Temmer et al., 2007), while the same harmonics have also been identified in auroral electrons (Emery et al., 2009). Love et al. (2012) made an analysis of the geomagnetic activity $a a$ index and concluded that the presence of high levels of occurrence of particularly the 9.0- and 6.7-day periods during the minimum of 23-24 is related to unusually low levels of sunspot numbers as well as the sectorial spherical harmonic structure of the solar magnetic field. The spectral analysis of daily-mean $K$ indices at LER, HER, and CNB revealed that solar minima during the period between 1960 and 2010 are characterized by the occurrence of the 4th, 3rd, and 2nd harmonics of the synodic solar-rotational period. In fact it is also revealed in this investigation, using a Lomb-Scargle spectral analysis, that the solar magnetic field is dominated by the 13.5-day period during solar minima. This can possibly be the result of the non-axisymmetric structure in the solar magnetic field during periods of low sunspot numbers, particular during the downward and minimum phase of cycle 23 , when the poloidal field is dominating (Love et al., 2012). It is also observed that the 27.0-day rotational period drops below the $95 \%$ confidence level during this period, which can be due to solar activity dominated to a large extent by solar winds originating in coronal holes, having a direct influence on the heliosphere and the ensuing geomagnetic activity as recorded by $K$ indices at observatories located at both high and mid-latitudes. Although data for CNB are only available for the period 1985-2010, spectrum analysis results obtained for this observatory are in line with what is observed at LER and HER for the same period.

Acknowledgements. Constructive and useful comments by two anonymous referees when evaluating this paper are gratefully acknowledged. The author would also like to thank the British Geological Survey and Geoscience Australia for making $K$ index data available, as well as the Wilcox Solar Observatory for solar magnetic field model information.

Topical Editor G. Balasis thanks J. Wanliss and one anonymous referee for their help in evaluating this paper.

\section{References}

Balasis, G., Daglis, I. A., Kapiris, P., Mandea, M., Vassiliadis, D., and Eftaxias, K.: From pre-storm activity to magnetic storms: a transition described in terms of fractal dynamics, Ann. Geophys., 24, 3557-3567, doi:10.5194/angeo-24-3557-2006, 2006.

Bartels, J.: Terrestrial magnetic activity and its relation to solar phenomenon, J. Geophys. Res., 37, 1-52, 1932.

Cliver, E. W.: Solar activity and geomagnetic storms: From M regions and flares to coronal holes and CMEs, Eos Trans. AGU, 76, 75-83, 1995.

Crooker, N. U., Feynman, J., and Gosling, J. T.: On the high correlation between long-term averages of solar wind speed and geomagnetic activity, J. Geophys. Res., 82, 1933-1937, 1977.

Daubechies, I.: Ten Lectures on Wavelets, Philadelphia: Society for Industrial and Applied Mathematics, 359 pp., 1992.

Emery, B. A., Richardson, I. G., Evans, D. S., and Rich, F. J.: Solar wind structure sources and periodicities of auroral electron power over three solar cycles, J. Atmos. Sol. Terr. Phys., 71, 1157-1175, 2009.

Gonzalez, A. L. C. and Gonzalez, W. D.: Periodicities in the interplanetary magnetic field polarity, J. Geophys. Res., 92, 43574375, 1987.

Gosling, J. T., McComas, D. J., Phillips, J. L., and Bame, S. J.: Geomagnetic activity associated with Earth passage of interplanetary shock disturbances and coronal mass ejections, J. Geophys. Res., 96, 7831-7839, 1991.

Katsavrias, C., Preka-Papadema, P., and Moussas, X.: Wavelet analysis on solar wind parameters and geomagnetic indices, Sol. Phys., 280, 623-640, doi:10.1007/s11207-012-0078-6, 2012.

Kojima, M. and Kakinuma, T.: Solar cycle dependence of global distribution of solar wind speed, Space Sci. Rev., 53, 173-222, 1990.

Legrand, J. P. and Simon, P. A.: A two-component solar cycle, Sol. Physics, 131, 187-209, 1991. 
Lei, J., Thayer, J. P., Forbes, J. M., Sutton, E. K., and Nerem, R. S.: Rotating solar coronal holes and periodic modulation of the upper atmosphere, Geophys. Res. Lett., 35, L10109, doi:10.1029/2008GL033875, 2008.

Lomb, N. R.: Least-squares frequency analysis of unequally spaced data, Astrophys. Spa. Sci., 39, 447-462, doi:10.1007/BF00648343, 1976.

Love, J. J., Rigler, E. J., and Gibson, S. E.: Geomagnetic detection of the sectorial solar magnetic field and the historical peculiarity of minimum 23-24, Geophys. Res. Lett., 39, L04102, doi:10.1029/2011GL050702, 2012.

Maunder, E. W.: Magnetic disturbances, 1882 to 1903, as recorded at the Royal Observatory, Greenwich, and their association with sunspots, Mon. Not. R. Astron. Soc. London, 65, 2, 1905.

Morlet, J., Arens, G., Forgeau, I., and Giard, D.: Wave propagation and sampling theory, Geophys., 47, 203-236, doi:10.1190/1.1441328, 1982.

Mursula, K.: Simultaneous occurrence of mid-term periodicities in solar wind speed, geomagnetic activity and cosmic rays, edited by: Kieda, D., Salamon, M., and Dingus, B., IUPAP Proceedings of the 26th International Cosmic Ray Conference 7, p. 126, 1999.

Mursula, K. and Zieger, B.: The 13.5 day periodicity in the Sun, solar wind, and geomagnetic activity: The last three solar cycles, AGU, 101, 27077-27090, 1996.

Mursula, K. and Zieger, B.: Solar excursion phases during the last 14 solar cycles, Geophys. Res. Lett., 25, 1851-1854, 1998.

Neupert, W. M. and Pizzo, V.: Solar coronal holes as sources of recurrent geomagnetic disturbances, J. Geophys. Res., 79, 37013709, 1974.
Press, W. H., Teukolsy, S. A., Vetterling, W. T., and Flannery, B. P.: Numerical Recipes in Fortran: The Art of Scientific Computing, Cambridge Univ. Press, UK, 963 pp., 1992.

Richardson, I. G., Cane, H. V., and Cliver, E. W.: Sources of geomagnetic storms for solar minimum and maximum conditions during 1972-2000, Geophys. Res. Lett., 28, 2569-2572, 2001.

Russell, C. T., Luhmann, J. G., and Jian, L. K.: How unprecedented a solar minimum?, Rev. Geophys., 48, RG2004, doi:10.1029/2009RG000316, 2010.

Scargle, J. D.: Studies in astronomical time series analysis II - Statistical aspects of spectral analysis of unevenly spaced data, Astrophys. J., 263, 835-853, doi:10.1086/160554, 1982.

Sheeley Jr., N. R., Harvey, J. W., and Feldman, W. C.: Coronal holes, solar wind streams, and recurrent geomagnetic disturbances 1973-1976, Sol. Phys., 49, 271-278, 1976.

Solanki, S. K., Inhester, B., and Schüssler, M.: The solar magnetic field, Rep. Prog. Phys., 69, 563-668, 2006.

Temmer, M., Vršnak, B., and Veronig, A. M.: Periodic appearance of coronal holes and the related variation of solar wind parameters, Sol. Phys., 241, 371-383, doi:10.1007/s11207-007-0336-1, 2007.

Torrence, C. and Compo, G. P.: A practical guide to wavelet analysis, Bull. Am. Meteorol. Soc., 79, 61-78, 1998.

Tsurutani, B. R., McPherron, R., Gonzalez, W., Lu, G., Sobral, J. H. A., and Gopalswamy, N.: Recurrent Magnetic Storms: Corotating Solar Wind Streams, Geophys. Monogr. Ser., 167, 340 pp., AGU, Washington, DC, 2006. 